\title{
Pythagorean Tuning: Pentatonic and Heptatonic Scale
}

\author{
Roland Coghetto \\ Rue de la Brasserie 5 \\ 7100 La Louvière, Belgium
}

Summary. In this article, using the Mizar system [3], 4, we define a structure [1, 6] in order to build a Pythagorean pentatonic scale and a Pythagorean heptatonic scale ${ }^{1}$ [5], 7].

MSC: 00A65 97M80 03B35

Keywords: music; Pythagorean tuning; pentatonic scale; heptatonic scale MML identifier: MUSIC_S1, version: 8.1.08 5.53.1335

\section{Preliminaries}

Now we state the proposition:

(1) Let us consider an object $r$. Then $r \in \mathbb{R}_{+\cup\{0\}} \backslash\{0\}$ if and only if $r$ is a positive real number.

Note that there exists a rational number which is positive. term

The functor $\mathbb{Q}_{+}$yielding a non empty subset of $\mathbb{R}_{+\cup\{0\}}$ is defined by the

(Def. 1) the set of all $r$ where $r$ is a positive rational number.

Now we state the propositions:

(2) Let us consider an object $r$. Then $r$ is an element of $\mathbb{Q}_{+}$if and only if $r$ is a positive rational number.

(3) $\mathbb{Q}_{+\cup\{0\}} \subseteq \mathbb{Q}$.

${ }^{1}$ https://en.wikipedia.org/wiki/Pythagorean_tuning 
The functor $\mathbb{R}_{+}$yielding a non empty subset of $\mathbb{R}_{+\cup\{0\}}$ is defined by the term

(Def. 2) $\mathbb{R}_{+\cup\{0\}} \backslash\{0\}$.

Now we state the propositions:

(4) $\mathbb{N}_{+} \subseteq \mathbb{Q}_{+}$.

(5) $\mathbb{N}_{+} \subseteq \mathbb{R}_{+}$. The theorem is a consequence of (1).

(6) $\mathbb{Q}_{+} \subseteq \mathbb{R}_{+}$. The theorem is a consequence of (2) and (1).

\section{Real Frequency}

We consider structures of music which extend 1-sorted structures and are systems

$$
\text { 〈a carrier, an equidistance, a Ratio〉 }
$$

where the carrier is a set, the equidistance is a relation between (the carrier) $\times$ (the carrier) and (the carrier $) \times($ the carrier $)$, the Ratio is a function from (the carrier $) \times($ the carrier $)$ into the carrier.

Let $S$ be a structure of music and $a, b, c, d$ be elements of $S$. We say that $\overline{a b} \cong \overline{c d}$ if and only if

(Def. 3) $\langle\langle a, b\rangle,\langle c, d\rangle\rangle \in$ the equidistance of $S$.

Let $x, y$ be elements of $\mathbb{R}_{+}$. The functor $\mathbb{R}$-ratio $(x, y)$ yielding an element of $\mathbb{R}_{+}$is defined by

(Def. 4) there exist positive real numbers $r, s$ such that $x=r$ and $s=y$ and it $=\frac{s}{r}$.

Now we state the proposition:

(7) Let us consider elements $a, b, c, d$ of $\mathbb{R}_{+}$. Then $\mathbb{R}$-ratio $(a, b)=\mathbb{R}$-ratio $(c, d)$ if and only if $\mathbb{R}$-ratio $(b, a)=\mathbb{R}$-ratio $(d, c)$.

The functor $\mathbb{R}$-ratio yielding a function from $\mathbb{R}_{+} \times \mathbb{R}_{+}$into $\mathbb{R}_{+}$is defined by

(Def. 5) for every element $x$ of $\mathbb{R}_{+} \times \mathbb{R}_{+}$, there exist elements $y, z$ of $\mathbb{R}_{+}$such that $x=\langle y, z\rangle$ and it $(x)=\mathbb{R}-\operatorname{ratio}(y, z)$.

The functor eq- $\mathbb{R}$-ratio yielding a relation between $\mathbb{R}_{+} \times \mathbb{R}_{+}$and $\mathbb{R}_{+} \times \mathbb{R}_{+}$ is defined by

(Def. 6) for every elements $x, y$ of $\mathbb{R}_{+} \times \mathbb{R}_{+},\langle x, y\rangle \in i t$ iff there exist elements $a, b, c, d$ of $\mathbb{R}_{+}$such that $x=\langle a, b\rangle$ and $y=\langle c, d\rangle$ and $\mathbb{R}$-ratio $(a, b)=$ $\mathbb{R}$-ratio $(c, d)$.

The functor $\mathbb{R}$-music yielding a structure of music is defined by the term

(Def. 7) $\left\langle\mathbb{R}_{+}\right.$, eq- $\mathbb{R}$-ratio, $\mathbb{R}$-ratio $\rangle$.

Now we state the propositions: 
(8) (i) $\mathbb{R}$-music is not empty, and

(ii) the carrier of $\mathbb{R}$-music $\subseteq \mathbb{R}_{+}$, and

(iii) for every elements $f_{1}, f_{2}, f_{3}, f_{4}$ of $\mathbb{R}$-music, $\overline{f_{1} f_{2}} \cong \overline{f_{3} f_{4}}$ iff (the Ratio of $\mathbb{R}$-music $)\left(f_{1}, f_{2}\right)=($ the Ratio of $\mathbb{R}$-music $)\left(f_{3}, f_{4}\right)$.

(9) Let us consider elements $f_{1}, f_{2}, f_{3}$ of $\mathbb{R}$-music. Suppose (the Ratio of $\mathbb{R}$-music $)\left(f_{1}, f_{2}\right)=($ the Ratio of $\mathbb{R}$-music $)\left(f_{1}, f_{3}\right)$. Then $f_{2}=f_{3}$.

(10) $\mathbb{N}_{+} \subseteq$ the carrier of $\mathbb{R}$-music.

(11) Let us consider an element fr of $\mathbb{R}$-music, and a non zero natural number $n$. Then there exists an element $h$ of $\mathbb{R}$-music such that $\langle f r, h\rangle \in[\langle 1, n\rangle]_{\alpha}$, where $\alpha$ is the equidistance of $\mathbb{R}$-music. The theorem is a consequence of (1) and (8).

(12) Let us consider elements $f_{1}, f_{2}, f_{3}$ of $\mathbb{R}$-music. Suppose (the Ratio of $\mathbb{R}$-music $)\left(f_{1}, f_{1}\right)=($ the Ratio of $\mathbb{R}$-music $)\left(f_{2}, f_{3}\right)$. Then $f_{2}=f_{3}$.

(13) Let us consider elements $f_{1}, f_{2}, f_{8}, f_{6}, f_{9}, f_{7}$ of $\mathbb{R}$-music, positive real numbers $r_{1}, r_{2}$, and non zero natural numbers $n, m$. Suppose $f_{8}=n \cdot r_{1}$ and $f_{6}=m \cdot r_{1}$ and $f_{9}=n \cdot r_{2}$ and $f_{7}=m \cdot r_{2}$. Then $\overline{f_{8} f_{6}} \cong \overline{f_{9} f_{7}}$. The theorem is a consequence of $(8)$.

(14) Let us consider elements $f_{1}, f_{2}, f_{3}, f_{4}$ of $\mathbb{R}$-music. Then (the Ratio of $\mathbb{R}$-music) $\left(f_{1}, f_{2}\right)=$ (the Ratio of $\mathbb{R}$-music) $\left(f_{3}, f_{4}\right)$ if and only if (the Ratio of $\mathbb{R}$-music $)\left(f_{2}, f_{1}\right)=($ the Ratio of $\mathbb{R}$-music $)\left(f_{4}, f_{3}\right)$. The theorem is a consequence of $(7)$.

\section{Rational Frequency}

Let $x, y$ be elements of $\mathbb{Q}_{+}$. The functor $\mathbb{Q}$-ratio $(x, y)$ yielding an element of $\mathbb{Q}_{+}$is defined by

(Def. 8) there exist positive rational numbers $r, s$ such that $x=r$ and $s=y$ and $i t=\frac{s}{r}$.

Now we state the proposition:

(15) Let us consider elements $a, b, c, d$ of $\mathbb{Q}_{+} \cdot$ Then $\mathbb{Q}$-ratio $(a, b)=\mathbb{Q}$-ratio $(c, d)$ if and only if $\mathbb{Q}$-ratio $(b, a)=\mathbb{Q}-\operatorname{ratio}(d, c)$.

The functor $\mathbb{Q}$-ratio yielding a function from $\mathbb{Q}_{+} \times \mathbb{Q}_{+}$into $\mathbb{Q}_{+}$is defined by

(Def. 9) for every element $x$ of $\mathbb{Q}_{+} \times \mathbb{Q}_{+}$, there exist elements $y, z$ of $\mathbb{Q}_{+}$such that $x=\langle y, z\rangle$ and $i t(x)=\mathbb{Q}$-ratio $(y, z)$.

The functor eq- $\mathbb{Q}$-ratio yielding a relation between $\mathbb{Q}_{+} \times \mathbb{Q}_{+}$and $\mathbb{Q}_{+} \times \mathbb{Q}_{+}$ is defined by 
(Def. 10) for every elements $x, y$ of $\mathbb{Q}_{+} \times \mathbb{Q}_{+},\langle x, y\rangle \in$ it iff there exist elements $a, b, c, d$ of $\mathbb{Q}_{+}$such that $x=\langle a, b\rangle$ and $y=\langle c, d\rangle$ and $\mathbb{Q}$-ratio $(a, b)=$ $\mathbb{Q}$-ratio $(c, d)$.

The functor $\mathbb{Q}$-music yielding a structure of music is defined by the term

(Def. 11) $\left\langle\mathbb{Q}_{+}\right.$, eq- $\mathbb{Q}$-ratio, $\mathbb{Q}$-ratio $\rangle$.

Now we state the propositions:

(16) (i) $\mathbb{Q}$-music is not empty, and

(ii) the carrier of $\mathbb{Q}$-music $\subseteq \mathbb{R}_{+}$, and

(iii) for every elements $f_{1}, f_{2}, f_{3}, f_{4}$ of $\mathbb{Q}$-music, $\overline{f_{1} f_{2}} \cong \overline{f_{3} f_{4}}$ iff (the Ratio of $\mathbb{Q}$-music $)\left(f_{1}, f_{2}\right)=($ the Ratio of $\mathbb{Q}$-music $)\left(f_{3}, f_{4}\right)$.

The theorem is a consequence of (6).

(17) Let us consider elements $f_{1}, f_{2}, f_{3}$ of $\mathbb{Q}$-music. Suppose (the Ratio of $\mathbb{Q}$-music $)\left(f_{1}, f_{2}\right)=($ the Ratio of $\mathbb{Q}$-music $)\left(f_{1}, f_{3}\right)$. Then $f_{2}=f_{3}$.

(18) $\mathbb{N}_{+} \subseteq$ the carrier of $\mathbb{Q}$-music.

(19) Let us consider an element fr of $\mathbb{Q}$-music, and a non zero natural number $n$. Then there exists an element $h$ of $\mathbb{Q}$-music such that $\langle f r, h\rangle \in[\langle 1, n\rangle]_{\alpha}$, where $\alpha$ is the equidistance of $\mathbb{Q}$-music. The theorem is a consequence of (2) and (16).

(20) Let us consider elements $f_{1}, f_{2}, f_{3}$ of $\mathbb{Q}$-music. Suppose (the Ratio of $\mathbb{Q}$-music $)\left(f_{1}, f_{1}\right)=($ the Ratio of $\mathbb{Q}$-music $)\left(f_{2}, f_{3}\right)$. Then $f_{2}=f_{3}$.

(21) Let us consider an element $f r$ of $\mathbb{Q}$-music. Then there exists a positive real number $r$ such that

(i) $f r=r$, and

(ii) for every non zero natural number $n, n \cdot r$ is an element of $\mathbb{Q}$-music.

The theorem is a consequence of (2).

(22) Let us consider elements $f_{1}, f_{2}, f_{8}, f_{6}, f_{9}, f_{7}$ of $\mathbb{Q}$-music, positive rational numbers $r_{1}, r_{2}$, and non zero natural numbers $n, m$. Suppose $f_{8}=n \cdot r_{1}$ and $f_{6}=m \cdot r_{1}$ and $f_{9}=n \cdot r_{2}$ and $f_{7}=m \cdot r_{2}$. Then $\overline{f_{8} f_{6}} \cong \overline{f_{9} f_{7}}$. The theorem is a consequence of (16).

(23) Let us consider elements $f_{1}, f_{2}, f_{3}, f_{4}$ of $\mathbb{Q}$-music. Then (the Ratio of $\mathbb{Q}$-music $)\left(f_{1}, f_{2}\right)=($ the Ratio of $\mathbb{Q}$-music $)\left(f_{3}, f_{4}\right)$ if and only if (the Ratio of $\mathbb{Q}$-music $)\left(f_{2}, f_{1}\right)=$ (the Ratio of $\mathbb{Q}$-music $)\left(f_{4}, f_{3}\right)$. The theorem is a consequence of (15). 


\section{Musical Structure and Some Axioms}

Let $S$ be a structure of music. We say that $S$ is satisfying real if and only if (Def. 12) the carrier of $S \subseteq \mathbb{R}_{+}$.

We say that $S$ is equidistant-ratio equivalent if and only if

(Def. 13) for every elements $f_{1}, f_{2}, f_{3}, f_{4}$ of $S, \overline{f_{1} f_{2}} \cong \overline{f_{3} f_{4}}$ iff (the Ratio of $S)\left(f_{1}, f_{2}\right)=($ the Ratio of $S)\left(f_{3}, f_{4}\right)$.

We say that $S$ is satisfying interval if and only if

(Def. 14) for every elements $f_{1}, f_{2}, f_{3}$ of $S$ such that (the Ratio of $\left.S\right)\left(f_{1}, f_{2}\right)=$ (the Ratio of $S)\left(f_{1}, f_{3}\right)$ holds $f_{2}=f_{3}$.

We say that $S$ is unison-ratio stable if and only if

(Def. 15) for every elements $f_{1}, f_{2}, f_{3}$ of $S$ such that (the Ratio of $\left.S\right)\left(f_{1}, f_{1}\right)=$ (the Ratio of $S)\left(f_{2}, f_{3}\right)$ holds $f_{2}=f_{3}$.

We say that $S$ is ratio symmetric if and only if

(Def. 16) for every elements $f_{1}, f_{2}, f_{3}, f_{4}$ of $S$, (the Ratio of $\left.S\right)\left(f_{1}, f_{2}\right)=$ (the Ratio of $S)\left(f_{3}, f_{4}\right)$ iff (the Ratio of $\left.S\right)\left(f_{2}, f_{1}\right)=$ (the Ratio of $\left.S\right)\left(f_{4}, f_{3}\right)$.

We say that $S$ is natural if and only if

(Def. 17) $\quad \mathbb{N}_{+} \subseteq$ the carrier of $S$.

We say that $S$ is harmonic closed if and only if

(Def. 18) for every element fr of $S$ and for every non zero natural number $n$, there exists an element $h$ of $S$ such that $\langle f r, h\rangle \in[\langle 1, n\rangle]_{\alpha}$, where $\alpha$ is the equidistance of $S$.

Note that there exists a structure of music which is harmonic closed, natural, ratio symmetric, unison-ratio stable, satisfying interval, equidistant-ratio equivalent, satisfying real, and non empty.

Let us note that the functor $\mathbb{R}$-music yields a harmonic closed, natural, ratio symmetric, unison-ratio stable, satisfying interval, equidistant-ratio equivalent, satisfying real, non empty structure of music. Observe that the functor $\mathbb{Q}$-music yields a harmonic closed, natural, ratio symmetric, unison-ratio stable, satisfying interval, equidistant-ratio equivalent, satisfying real, non empty structure of music. Now we state the propositions:

(24) Let us consider a natural structure of music $S$. Then every non zero natural number is an element of $S$.

(25) Let us consider an equidistant-ratio equivalent structure of music $M$, and elements $a, b$ of $M$. Then $\overline{a b} \cong \overline{a b}$.

(26) Let us consider an equidistant-ratio equivalent structure of music $M$, and elements $a, b, c, d$ of $M$. Then $\overline{a b} \cong \overline{c d}$ if and only if $\overline{c d} \cong \overline{a b}$. 
(27) Let us consider an equidistant-ratio equivalent structure of music $M$, and elements $a, b, c, d, e, f$ of $M$. Suppose $\overline{a b} \cong \overline{c d}$ and $\overline{c d} \cong \overline{e f}$. Then $\overline{a b} \cong \overline{e f}$.

(28) Let us consider a satisfying interval, equidistant-ratio equivalent structure of music $S$, and elements $a, b, c$ of $S$. Then $\overline{a b} \cong \overline{a c}$ if and only if $b=c$. The theorem is a consequence of (25).

From now on $M$ denotes an equidistant-ratio equivalent structure of music and $a, b, c, d, e, f$ denote elements of $M$.

Now we state the propositions:

(29) $\overline{a a} \cong \overline{a a}$.

(30) The equidistance of $M$ is reflexive in (the carrier of $M) \times($ the carrier of $M)$. The theorem is a consequence of $(25)$.

(31) Suppose $M$ is not empty. Then

(i) the equidistance of $M$ is reflexive, and

(ii) field(the equidistance of $M)=($ the carrier of $M) \times($ the carrier of $M)$.

The theorem is a consequence of (30).

(32) The equidistance of $M$ is symmetric in (the carrier of $M) \times($ the carrier of $M)$. The theorem is a consequence of (26).

(33) The equidistance of $M$ is transitive in (the carrier of $M) \times($ the carrier of $M)$. The theorem is a consequence of (27).

(34) The equidistance of $M$ is an equivalence relation of (the carrier of $M) \times$ (the carrier of $M$ ). The theorem is a consequence of (30), (32), and (33).

(35) Let us consider a ratio symmetric, equidistant-ratio equivalent structure of music $M$, and elements $a, b, c, d$ of $M$. Then $\overline{a b} \cong \overline{c d}$ if and only if $\overline{b a} \cong \overline{d c}$.

(36) Let us consider a unison-ratio stable, equidistant-ratio equivalent structure of music $S$, and elements $a, b, c$ of $S$. If $\overline{a a} \cong \overline{b c}$, then $b=c$.

Let $S$ be a natural, satisfying interval, harmonic closed, equidistant-ratio equivalent structure of music, $f r$ be an element of $S$, and $n$ be a non zero natural number. The $n$-harmonic of $f r$ in $S$ yielding an element of $S$ is defined by

(Def. 19) $\langle f r, i t\rangle \in[\langle 1, n\rangle]_{\alpha}$, where $\alpha$ is the equidistance of $S$.

We say that $S$ is harmonic linear if and only if

(Def. 20) for every element $f r$ of $S$ and for every non zero natural number $n$, there exists a positive real number $f$ such that $f r=f$ and the $n$-harmonic of $f r$ in $S=n \cdot f$.

Now we state the propositions: 
(37) $\mathbb{R}$-music is harmonic linear. The theorem is a consequence of (1) and (24).

(38) $\mathbb{Q}$-music is harmonic linear. The theorem is a consequence of (2) and (24).

One can check that there exists a harmonic closed, natural, ratio symmetric, unison-ratio stable, satisfying interval, equidistant-ratio equivalent, satisfying real, non empty structure of music which is harmonic linear.

One can check that the functor $\mathbb{R}$-music yields a harmonic linear, harmonic closed, natural, ratio symmetric, unison-ratio stable, satisfying interval, equidistant-ratio equivalent, satisfying real, non empty structure of music. Let us note that the functor $\mathbb{Q}$-music yields a harmonic linear, harmonic closed, natural, ratio symmetric, unison-ratio stable, satisfying interval, equidistantratio equivalent, satisfying real, non empty structure of music.

Let $M$ be a harmonic closed, natural, satisfying interval, equidistant-ratio equivalent structure of music. We say that $M$ is harmonic stable if and only if

(Def. 21) for every elements $f_{1}, f_{2}$ of $M$ and for every non zero natural numbers $n, m$, the $n$-harmonic of $f_{1}$ in $M$ the $m$-harmonic of $f_{1}$ in $M \cong$ the $n$-harmonic of $f_{2}$ in $M$ the $m$-harmonic of $f_{2}$ in $M$.

Now we state the propositions:

(39) $\mathbb{R}$-music is harmonic stable. The theorem is a consequence of (1) and (13).

(40) $\mathbb{Q}$-music is harmonic stable. The theorem is a consequence of (2) and $(22)$.

Observe that there exists a harmonic linear, harmonic closed, natural, ratio symmetric, unison-ratio stable, satisfying interval, equidistant-ratio equivalent, satisfying real, non empty structure of music which is harmonic stable.

One can verify that the functor $\mathbb{R}$-music yields a harmonic stable, harmonic linear, harmonic closed, natural, ratio symmetric, unison-ratio stable, satisfying interval, equidistant-ratio equivalent, satisfying real, non empty structure of music. Observe that the functor $\mathbb{Q}$-music yields a harmonic stable, harmonic linear, harmonic closed, natural, ratio symmetric, unison-ratio stable, satisfying interval, equidistant-ratio equivalent, satisfying real, non empty structure of music.

Let $M$ be a harmonic closed, natural, satisfying interval, equidistant-ratio equivalent structure of music and $f r$ be an element of $M$. The functors: the set of unison of $f r$ in $M$, the set of octave of $f r$ in $M$, the set of fifth of $f r$ in $M$, the set of fourth of $f r$ in $M$, and the set of major sixth of $f r$ in $M$ yielding subsets of (the carrier of $M) \times($ the carrier of $M$ ) are defined by terms 
(Def. 22) [〈the 1-harmonic of $f r$ in $M$, the 1-harmonic of $f r$ in $M\rangle]_{\alpha}$, where $\alpha$ is the equidistance of $M$,

(Def. 23) [〈the 1-harmonic of $f r$ in $M$, the 2-harmonic of $f r$ in $M\rangle]_{\alpha}$, where $\alpha$ is the equidistance of $M$,

(Def. 24) [〈the 2-harmonic of $f r$ in $M$, the 3-harmonic of $f r$ in $M\rangle]_{\alpha}$, where $\alpha$ is the equidistance of $M$,

(Def. 25) [〈the 3-harmonic of $f r$ in $M$, the 4-harmonic of $f r$ in $M\rangle]_{\alpha}$, where $\alpha$ is the equidistance of $M$,

(Def. 26) [〈the 3-harmonic of $f r$ in $M$, the 5-harmonic of $f r$ in $M\rangle]_{\alpha}$, where $\alpha$ is the equidistance of $M$,

respectively. The functors: the set of major third of $f r$ in $M$, the set of minor third of $f r$ in $M$, the set of minor sixth of $f r$ in $M$, the set of major tone of $f r$ in $M$, and the set of minor tone of $f r$ in $M$ yielding subsets of (the carrier of $M) \times($ the carrier of $M)$ are defined by terms

(Def. 27) [〈the 4-harmonic of $f r$ in $M$, the 5-harmonic of $f r$ in $M\rangle]_{\alpha}$, where $\alpha$ is the equidistance of $M$,

(Def. 28) $\quad[\langle\text { the } 5 \text {-harmonic of } f r \text { in } M \text {, the 6-harmonic of } f r \text { in } M\rangle]_{\alpha}$, where $\alpha$ is the equidistance of $M$,

(Def. 29) [〈the 5-harmonic of $f r$ in $M$, the 8-harmonic of $f r$ in $M\rangle]_{\alpha}$, where $\alpha$ is the equidistance of $M$,

(Def. 30) [〈the 8-harmonic of $f r$ in $M$, the 9-harmonic of $f r$ in $M\rangle]_{\alpha}$, where $\alpha$ is the equidistance of $M$,

(Def. 31) [〈the 9-harmonic of $f r$ in $M$, the 10-harmonic of $f r$ in $M\rangle]_{\alpha}$, where $\alpha$ is the equidistance of $M$,

respectively. The functors: the set of unison of $M$, the set of octave of $M$, the set of fifth of $M$, the set of fourth of $M$, and the set of major sixth of $M$ yielding subsets of (the carrier of $M) \times($ the carrier of $M$ ) are defined by terms

(Def. 32) $[\langle 1,1\rangle]_{\alpha}$, where $\alpha$ is the equidistance of $M$,

(Def. 33) $[\langle 1,2\rangle]_{\alpha}$, where $\alpha$ is the equidistance of $M$,

(Def. 34) $[\langle 2,3\rangle]_{\alpha}$, where $\alpha$ is the equidistance of $M$,

(Def. 35) $[\langle 3,4\rangle]_{\alpha}$, where $\alpha$ is the equidistance of $M$,

(Def. 36) $[\langle 3,5\rangle]_{\alpha}$, where $\alpha$ is the equidistance of $M$,

respectively. The functors: the set of major third of $M$, the set of minor third of $M$, the set of minor sixth of $M$, the set of major tone of $M$, and the set of minor tone of $M$ yielding subsets of (the carrier of $M) \times($ the carrier of $M)$ are defined by terms

(Def. 37) $[\langle 4,5\rangle]_{\alpha}$, where $\alpha$ is the equidistance of $M$, 
(Def. 38) $[\langle 5,6\rangle]_{\alpha}$, where $\alpha$ is the equidistance of $M$,

(Def. 39) $[\langle 5,8\rangle]_{\alpha}$, where $\alpha$ is the equidistance of $M$,

(Def. 40) $[\langle 8,9\rangle]_{\alpha}$, where $\alpha$ is the equidistance of $M$,

(Def. 41) $[\langle 9,10\rangle]_{\alpha}$, where $\alpha$ is the equidistance of $M$,

respectively. Let $S$ be a harmonic closed, natural, satisfying interval, equidistantratio equivalent structure of music. We say that $S$ is fifth constructible if and only if

(Def. 42) for every element fr of $S$, there exists an element $q$ of $S$ such that $\langle f r$, $q\rangle \in$ the set of fifth of $S$.

Now we state the propositions:

(41) Let us consider an element $f r$ of $\mathbb{R}$-music. Then there exist positive real numbers $f, q_{1}$ such that

(i) $f=f r$, and

(ii) $q_{1}=\frac{(3 \text { qua real number })}{2} \cdot f$, and

(iii) $\left\langle f, q_{1}\right\rangle \in$ the set of fifth of $\mathbb{R}$-music.

The theorem is a consequence of (1) and (24).

(42) $\mathbb{R}$-music is fifth constructible. The theorem is a consequence of (41) and (1).

(43) Let us consider an element $f r$ of $\mathbb{Q}$-music. Then there exist positive rational numbers $f, q_{1}$ such that

(i) $f=f r$, and

(ii) $q_{1}=\frac{(3 \text { qua rational number })}{2} \cdot f$, and

(iii) $\left\langle f, q_{1}\right\rangle \in$ the set of fifth of $\mathbb{Q}$-music.

The theorem is a consequence of (2) and (24).

(44) $\mathbb{Q}$-music is fifth constructible. The theorem is a consequence of (43) and (2).

Let us observe that there exists a harmonic stable, harmonic linear, harmonic closed, natural, ratio symmetric, unison-ratio stable, satisfying interval, equidistant-ratio equivalent, satisfying real, non empty structure of music which is fifth constructible.

Let us note that the functor $\mathbb{R}$-music yields a fifth constructible, harmonic stable, harmonic linear, harmonic closed, natural, ratio symmetric, unisonratio stable, satisfying interval, equidistant-ratio equivalent, satisfying real, non empty structure of music. Let us note that the functor $\mathbb{Q}$-music yields a fifth constructible, harmonic stable, harmonic linear, harmonic closed, natural, ratio symmetric, unison-ratio stable, satisfying interval, equidistant-ratio equivalent, satisfying real, non empty structure of music. 
Let $M$ be a fifth constructible, harmonic closed, natural, satisfying interval, equidistant-ratio equivalent structure of music and $f r$ be an element of $M$. The fifth of $f r$ in $M$ yielding an element of $M$ is defined by

(Def. 43) $\langle f r, i t\rangle \in$ the set of fifth of $M$.

Now we state the propositions:

(45) Let us consider a fifth constructible, harmonic stable, harmonic linear, harmonic closed, natural, satisfying interval, equidistant-ratio equivalent structure of music $M$, and an element $f r$ of $M$. Then the set of fifth of $f r$ in $M=$ the set of fifth of $M$. The theorem is a consequence of (24) and (27).

(46) Let us consider an element $f r$ of $\mathbb{R}$-music. Then there exists a positive real number $f$ such that

(i) $f r=f$, and

(ii) the fifth of $f r$ in $\mathbb{R}$-music $=\frac{(3 \text { qua real number })}{2} \cdot f$.

The theorem is a consequence of (1) and (41).

(47) Let us consider an element fr of $\mathbb{Q}$-music. Then there exists a positive rational number $f$ such that

(i) $f r=f$, and

(ii) the fifth of $f r$ in $\mathbb{Q}$-music $=\frac{(3 \text { qua rational number })}{2} \cdot f$.

The theorem is a consequence of (2) and (43).

Let $M$ be a fifth constructible, harmonic closed, natural, satisfying interval, equidistant-ratio equivalent structure of music. We say that $M$ is classical fifth if and only if

(Def. 44) for every element $f r$ of $M$, there exists a positive real number $f$ such that $f r=f$ and the fifth of $f r$ in $M=\frac{(3 \text { qua real number })}{2} \cdot f$.

One can verify that there exists a fifth constructible, harmonic stable, harmonic linear, harmonic closed, natural, ratio symmetric, unison-ratio stable, satisfying interval, equidistant-ratio equivalent, satisfying real, non empty structure of music which is classical fifth.

One can verify that the functor $\mathbb{R}$-music yields a classical fifth, fifth constructible, harmonic stable, harmonic linear, harmonic closed, natural, ratio symmetric, unison-ratio stable, satisfying interval, equidistant-ratio equivalent, satisfying real, non empty structure of music.

One can check that the functor $\mathbb{Q}$-music yields a classical fifth, fifth constructible, harmonic stable, harmonic linear, harmonic closed, natural, ratio symmetric, unison-ratio stable, satisfying interval, equidistant-ratio equivalent, satisfying real, non empty structure of music. 


\section{HARMONIC}

Now we state the propositions:

(48) Let us consider a harmonic closed, natural, unison-ratio stable, satisfying interval, equidistant-ratio equivalent structure of music $M$, and an element $f r$ of $M$. Then the 1-harmonic of $f r$ in $M=f r$. The theorem is a consequence of (36).

(49) Let us consider a harmonic stable, harmonic closed, natural, unisonratio stable, satisfying interval, equidistant-ratio equivalent structure of music $M$, and elements $a, b$ of $M$. Then $\overline{a a} \cong \overline{b b}$. The theorem is a consequence of (48).

(50) Let us consider a harmonic stable, harmonic linear, harmonic closed, natural, unison-ratio stable, satisfying interval, equidistant-ratio equivalent structure of music $M$, and an element $f r$ of $M$. Then the set of octave of $\mathrm{fr}$ in $M=$ the set of octave of $M$. The theorem is a consequence of (48), (27), and (24).

(51) Let us consider a fifth constructible, harmonic closed, natural, satisfying interval, equidistant-ratio equivalent, non empty structure of music $M$, and an element $f r$ of $M$. Then there exists a sequence $s_{11}$ of $M$ such that

(i) $s_{11}(0)=f r$, and

(ii) for every natural number $n,\left\langle s_{11}(n), s_{11}(n+1)\right\rangle \in$ the set of fifth of $M$.

Proof: Define $\mathcal{P}$ [set, set, set $] \equiv$ there exist positive real numbers $x, y$ such that $\left\langle \$_{2}, \$_{3}\right\rangle \in$ the set of fifth of $M$. For every natural number $n$ and for every element $x$ of $M$, there exists an element $y$ of $M$ such that $\mathcal{P}[n, x, y]$. Consider $s_{11}$ being a sequence of $M$ such that $s_{11}(0)=f r$ and for every natural number $n, \mathcal{P}\left[n, s_{11}(n), s_{11}(n+1)\right]$.

Let $M$ be a structure of music and $a, b, c$ be elements of $M$. We say that $b$ is between $a$ and $c$ if and only if

(Def. 45) there exist positive real numbers $r_{1}, r_{2}, r_{3}$ such that $a=r_{1}$ and $b=r_{2}$ and $c=r_{3}$ and $r_{1} \leqslant r_{2}<r_{3}$.

Let $S$ be a harmonic closed, natural, satisfying interval, equidistant-ratio equivalent structure of music. We say that $S$ is octave constructible if and only if

(Def. 46) for every element $f r$ of $S$, there exists an element $o$ of $S$ such that $\langle f r$, o) $\in$ the set of octave of $S$.

Now we state the propositions: 
(52) Let us consider an element fr of $\mathbb{R}$-music. Then there exist positive real numbers $f, q_{1}$ such that

(i) $f=f r$, and

(ii) $q_{1}=2 \cdot f$, and

(iii) $\left\langle f, q_{1}\right\rangle \in$ the set of octave of $\mathbb{R}$-music.

The theorem is a consequence of (1) and (24).

(53) $\mathbb{R}$-music is octave constructible. The theorem is a consequence of (52) and (1).

(54) Let us consider an element $f r$ of $\mathbb{Q}$-music. Then there exist positive rational numbers $f, q_{1}$ such that

(i) $f=f r$, and

(ii) $q_{1}=2 \cdot f$, and

(iii) $\left\langle f, q_{1}\right\rangle \in$ the set of octave of $\mathbb{Q}$-music.

The theorem is a consequence of (2) and (24).

(55) $\mathbb{Q}$-music is octave constructible. The theorem is a consequence of (54) and (2).

Let us note that there exists a classical fifth, fifth constructible, harmonic stable, harmonic linear, harmonic closed, natural, ratio symmetric, unisonratio stable, satisfying interval, equidistant-ratio equivalent, satisfying real, non empty structure of music which is octave constructible.

Let us observe that the functor $\mathbb{R}$-music yields an octave constructible, classical fifth, fifth constructible, harmonic stable, harmonic linear, harmonic closed, natural, ratio symmetric, unison-ratio stable, satisfying interval, equidistant-ratio equivalent, satisfying real, non empty structure of music. Let us note that the functor $\mathbb{Q}$-music yields an octave constructible, classical fifth, fifth constructible, harmonic stable, harmonic linear, harmonic closed, natural, ratio symmetric, unison-ratio stable, satisfying interval, equidistantratio equivalent, satisfying real, non empty structure of music.

Let $M$ be an octave constructible, harmonic closed, natural, satisfying interval, equidistant-ratio equivalent structure of music and $f r$ be an element of $M$. The octave of $f r$ in $M$ yielding an element of $M$ is defined by

(Def. 47) $\langle f r, i t\rangle \in$ the set of octave of $M$.

Let $M$ be a satisfying real, non empty structure of music and $r$ be an element of $M$. The functor ${ }^{{ }} r$ yielding a positive real number is defined by the term (Def. 48) $r$. 
Let $M$ be an octave constructible, harmonic closed, natural, satisfying interval, equidistant-ratio equivalent structure of music. We say that $M$ is classical octave if and only if

(Def. 49) for every element $f r$ of $M$, there exists a positive real number $f$ such that $f r=f$ and the octave of $f r$ in $M=2 \cdot f$.

Now we state the propositions:

(56) $\mathbb{R}$-music is classical octave. The theorem is a consequence of (52) and (1).

(57) $\mathbb{Q}$-music is classical octave. The theorem is a consequence of (54) and (2).

One can verify that there exists an octave constructible, classical fifth, fifth constructible, harmonic stable, harmonic linear, harmonic closed, natural, ratio symmetric, unison-ratio stable, satisfying interval, equidistant-ratio equivalent, satisfying real, non empty structure of music which is classical octave.

Observe that the functor $\mathbb{R}$-music yields a classical octave, octave constructible, classical fifth, fifth constructible, harmonic stable, harmonic linear, harmonic closed, natural, ratio symmetric, unison-ratio stable, satisfying interval, equidistant-ratio equivalent, satisfying real, non empty structure of music. Observe that the functor $\mathbb{Q}$-music yields a classical octave, octave constructible, classical fifth, fifth constructible, harmonic stable, harmonic linear, harmonic closed, natural, ratio symmetric, unison-ratio stable, satisfying interval, equidistant-ratio equivalent, satisfying real, non empty structure of music.

Let $M$ be an octave constructible, harmonic closed, natural, satisfying interval, equidistant-ratio equivalent structure of music. We say that $M$ is octave descending constructible if and only if

(Def. 50) for every element $f r$ of $M$, there exists an element $o$ of $M$ such that $\langle o$, $f r\rangle \in$ the set of octave of $M$.

Now we state the propositions:

(58) Let us consider an element $f r$ of $\mathbb{R}$-music. Then there exist positive real numbers $f, q_{1}$ such that

(i) $f=f r$, and

(ii) $q_{1}=\frac{(1 \text { qua real number })}{2} \cdot f$, and

(iii) $\left\langle q_{1}, f\right\rangle \in$ the set of octave of $\mathbb{R}$-music.

The theorem is a consequence of (1), (24), and (35).

(59) $\mathbb{R}$-music is octave descending constructible. The theorem is a consequence of (58) and (1). 
(60) Let us consider an element fr of $\mathbb{Q}$-music. Then there exist positive rational numbers $f, q_{1}$ such that

(i) $f=f r$, and

(ii) $q_{1}=\frac{(1 \text { qua rational number })}{2} \cdot f$, and

(iii) $\left\langle q_{1}, f\right\rangle \in$ the set of octave of $\mathbb{Q}$-music.

The theorem is a consequence of (2), (24), and (35).

(61) $\mathbb{Q}$-music is octave descending constructible. The theorem is a consequence of (60) and (2).

One can verify that there exists a classical octave, octave constructible, classical fifth, fifth constructible, harmonic stable, harmonic linear, harmonic closed, natural, ratio symmetric, unison-ratio stable, satisfying interval, equidistant-ratio equivalent, satisfying real, non empty structure of music which is octave descending constructible.

One can verify that the functor $\mathbb{R}$-music yields an octave descending constructible, classical octave, octave constructible, classical fifth, fifth constructible, harmonic stable, harmonic linear, harmonic closed, natural, ratio symmetric, unison-ratio stable, satisfying interval, equidistant-ratio equivalent, satisfying real, non empty structure of music. Note that the functor $\mathbb{Q}$-music yields an octave descending constructible, classical octave, octave constructible, classical fifth, fifth constructible, harmonic stable, harmonic linear, harmonic closed, natural, ratio symmetric, unison-ratio stable, satisfying interval, equidistant-ratio equivalent, satisfying real, non empty structure of music.

Let $M$ be an octave descending constructible, octave constructible, fifth constructible, harmonic closed, natural, ratio symmetric, satisfying interval, equidistant-ratio equivalent structure of music and $f r$ be an element of $M$. The octave descending of $f r$ in $M$ yielding an element of $M$ is defined by

(Def. 51) $\langle i t, f r\rangle \in$ the set of octave of $M$.

Now we state the propositions:

(62) Let us consider an octave descending constructible, classical octave, octave constructible, fifth constructible, harmonic closed, natural, ratio symmetric, satisfying interval, equidistant-ratio equivalent, satisfying real, non empty structure of music $M$, and an element $f r$ of $M$. Then there exists a positive real number $r$ such that

(i) $f r=r$, and

(ii) the octave descending of $f r$ in $M=\frac{r}{2}$.

The theorem is a consequence of (1). 
(63) Let us consider classical octave, octave constructible, classical fifth, fifth constructible, harmonic closed, natural, satisfying interval, equidistantratio equivalent structures of music $M_{1}, M_{2}$, an element $f_{1}$ of $M_{1}$, and an element $f_{2}$ of $M_{2}$. Suppose $f_{1}=f_{2}$. Then

(i) the fifth of $f_{1}$ in $M_{1}=$ the fifth of $f_{2}$ in $M_{2}$, and

(ii) the octave of $f_{1}$ in $M_{1}=$ the octave of $f_{2}$ in $M_{2}$.

(64) Let us consider octave descending constructible, classical octave, octave constructible, fifth constructible, harmonic closed, natural, ratio symmetric, satisfying interval, equidistant-ratio equivalent, satisfying real, non empty structures of music $M_{1}, M_{2}$, an element $f r_{1}$ of $M_{1}$, and an element $f r_{2}$ of $M_{2}$. Suppose $f r_{1}=f r_{2}$. Then the octave descending of $f r_{1}$ in $M_{1}=$ the octave descending of $f r_{2}$ in $M_{2}$. The theorem is a consequence of $(62)$.

Let $M$ be an octave descending constructible, octave constructible, fifth constructible, harmonic closed, natural, ratio symmetric, satisfying interval, equidistant-ratio equivalent structure of music and $f_{10}, f r$ be elements of $M$. The reduct fifth of the $f r$ with fundamental frequency $f_{10}$ in $M$ yielding an element of $M$ is defined by the term

(Def. 52) $\left\{\begin{array}{l}\text { the fifth of } f r \text { in } M \text {, if the fifth of } f r \text { in } M \text { is between } f_{10} \text { and the } \\ \text { octave of } f_{10} \text { in } M, \\ \text { the octave descending of (the fifth of } f r \text { in } M \text { ) in } M \text {, otherwise. }\end{array}\right.$

Now we state the propositions:

(65) Let us consider octave descending constructible, classical octave, octave constructible, classical fifth, fifth constructible, harmonic closed, natural, ratio symmetric, satisfying interval, equidistant-ratio equivalent, satisfying real, non empty structures of music $M_{1}, M_{2}$, elements $f r_{1}, f_{11}$ of $M_{1}$, and elements $f r_{2}, f_{12}$ of $M_{2}$. Suppose $f r_{1}=f r_{2}$ and $f_{11}=f_{12}$. Then the reduct fifth of the $f r_{1}$ with fundamental frequency $f_{11}$ in $M_{1}=$ the reduct fifth of the $f r_{2}$ with fundamental frequency $f_{12}$ in $M_{2}$. The theorem is a consequence of (63) and (64).

(66) Let us consider a classical fifth, fifth constructible, harmonic closed, natural, satisfying interval, equidistant-ratio equivalent structure of music $M$, and an element $f r$ of $M$. Then there exist positive real numbers $r, s$ such that

(i) $r=f r$, and

(ii) $s=\frac{(3 \text { qua real number })}{2} \cdot r$, and

(iii) the fifth of $f r$ in $M=s$. 
(67) Let us consider an octave descending constructible, classical octave, octave constructible, classical fifth, fifth constructible, harmonic closed, natural, satisfying interval, equidistant-ratio equivalent structure of music $M$, and elements $f_{10}, f r$ of $M$. Suppose $f r$ is between $f_{10}$ and the octave of $f_{10}$ in $M$. Then there exist positive real numbers $r_{1}, r_{2}, r_{3}$ such that

(i) $f_{10}=r_{1}$, and

(ii) $f r=r_{2}$, and

(iii) the octave of $f_{10}$ in $M=2 \cdot r_{1}$, and

(iv) $r_{1} \leqslant r_{2} \leqslant 2 \cdot r_{1}$.

(68) Let us consider an octave descending constructible, classical octave, octave constructible, classical fifth, fifth constructible, harmonic closed, natural, ratio symmetric, satisfying interval, equidistant-ratio equivalent, satisfying real, non empty structure of music $M$, and elements $f_{10}$, $f r$ of $M$. Suppose $f r$ is between $f_{10}$ and the octave of $f_{10}$ in $M$. Then the reduct fifth of the $f r$ with fundamental frequency $f_{10}$ in $M$ is between $f_{10}$ and the octave of $f_{10}$ in $M$. The theorem is a consequence of (67) and (62).

A space of music is an octave descending constructible, classical octave, octave constructible, classical fifth, fifth constructible, harmonic stable, harmonic linear, harmonic closed, natural, ratio symmetric, unison-ratio stable, satisfying interval, equidistant-ratio equivalent, satisfying real, non empty structure of music. Now we state the propositions:

(69) $\mathbb{R}$-music is a space of music.

(70) $\mathbb{Q}$-music is a space of music.

\section{Spiral of FifthS}

Now we state the proposition:

(71) Let us consider an octave descending constructible, octave constructible, fifth constructible, harmonic closed, natural, ratio symmetric, satisfying interval, equidistant-ratio equivalent, non empty structure of music $M$, and elements $f_{10}$, fr of $M$. Then there exists a sequence $s_{11}$ of $M$ such that

(i) $s_{11}(0)=f r$, and

(ii) for every natural number $n, s_{11}(n+1)=$ the reduct fifth of the $s_{11}(n)$ with fundamental frequency $f_{10}$ in $M$. 
Proof: Define $\mathcal{P}$ [set, set, set] $\equiv$ there exist elements $x, y$ of $M$ such that $x=\$_{2}$ and $y=\$_{3}$ and $y=$ the reduct fifth of the $x$ with fundamental frequency $f_{10}$ in $M$. For every natural number $n$ and for every element $x$ of $M$, there exists an element $y$ of $M$ such that $\mathcal{P}[n, x, y]$. Consider $s_{11}$ being a sequence of $M$ such that $s_{11}(0)=f r$ and for every natural number $n, \mathcal{P}\left[n, s_{11}(n), s_{11}(n+1)\right]$.

Let $M$ be an octave descending constructible, octave constructible, fifth constructible, harmonic closed, natural, ratio symmetric, satisfying interval, equidistant-ratio equivalent, non empty structure of music and $f_{10}, f r$ be elements of $M$. The spiral of fifths of $f r$ with fundamental frequency $f_{10}$ in $M$ yielding a sequence of $M$ is defined by

(Def. 53) $i t(0)=f r$ and for every natural number $n$, it $(n+1)=$ the reduct fifth of the it $(n)$ with fundamental frequency $f_{10}$ in $M$.

From now on $M$ denotes an octave descending constructible, classical octave, octave constructible, classical fifth, fifth constructible, harmonic closed, natural, ratio symmetric, satisfying interval, equidistant-ratio equivalent, satisfying real, non empty structure of music and $f_{10}, f r$ denote elements of $M$.

Now we state the propositions:

(72) Suppose $f r$ is between $f_{10}$ and the octave of $f_{10}$ in $M$. Let us consider a natural number $n$. Then (the spiral of fifths of $f r$ with fundamental frequency $f_{10}$ in $\left.M\right)(n)$ is between $f_{10}$ and the octave of $f_{10}$ in $M$. Proof: Define $\mathcal{P}$ [natural number] $\equiv$ (the spiral of fifths of $f r$ with fundamental frequency $f_{10}$ in $\left.M\right)\left(\$_{1}\right)$ is between $f_{10}$ and the octave of $f_{10}$ in $M$. For every natural number $k$ such that $\mathcal{P}[k]$ holds $\mathcal{P}[k+1]$. For every natural number $k, \mathcal{P}[k]$ from $[2$, Sch. 2].

(73) (The spiral of fifths of $f_{10}$ with fundamental frequency $f_{10}$ in $\left.M\right)(1)=$ $\frac{(3 \text { qua real number })}{2} \cdot\left({ }^{@} f_{10}\right)$. The theorem is a consequence of $(66)$.

(74) (The spiral of fifths of $f_{10}$ with fundamental frequency $f_{10}$ in $\left.M\right)(2)=$ $\frac{(9 \text { qua real number })}{8} \cdot\left({ }^{@} f_{10}\right)$. The theorem is a consequence of $(73),(66)$, and (62).

(75) (The spiral of fifths of $f_{10}$ with fundamental frequency $f_{10}$ in $\left.M\right)(3)=$ $\frac{(27 \text { qua real number) }}{16} \cdot\left({ }^{@} f_{10}\right)$. The theorem is a consequence of $(74)$ and $(66)$.

(76) (The spiral of fifths of $f_{10}$ with fundamental frequency $f_{10}$ in $\left.M\right)(4)=$ $\frac{\text { (81 qua real number) }}{64} \cdot\left({ }^{@} f_{10}\right)$. The theorem is a consequence of $(75),(66)$, and (62).

(77) (The spiral of fifths of $f_{10}$ with fundamental frequency $f_{10}$ in $\left.M\right)(5)=$ $\frac{(243 \text { qua real number })}{128} \cdot\left({ }^{@} f_{10}\right)$. The theorem is a consequence of $(76)$ and $(66)$.

$\frac{\varrho(\text { the spiral of fifths of } f r \text { with fundamental frequency } f r \text { in } M)(2)}{{ }^{\circ} f r}=$ 
$\frac{3 \cdot 3 \text { qua real number }}{2 \cdot 2 \cdot 2}$. The theorem is a consequence of $(74)$.

(79)

@ (the spiral of fifths of $f r$ with fundamental frequency $f r$ in $M)(4)$

$\frac{3 \cdot 3 \text { qua real number }}{2 \cdot 2 \cdot 2}$. The theorem is a consequence of (74) and (76).

(80) $\frac{@ \text { (the spiral of fifths of } f r \text { with fundamental frequency } f r \text { in } M)(1)}{@(\text { the spiral of fifths of } f r \text { with fundamental frequency } f r \text { in } M)(4)}=$

$\frac{32 \text { qua real number }}{27}$. The theorem is a consequence of (73) and (76).

(81) $\frac{@ \text { (the spiral of fifths of } f r \text { with fundamental frequency } f r \text { in } M)(3)}{@(\text { the spiral of fifths of } f r \text { with fundamental frequency } f r \text { in } M)(1)}=$

$\frac{9 \text { qua real number }}{8}$. The theorem is a consequence of (73) and (75).

(82)

@ (the octave of $f r$ in $M$ )

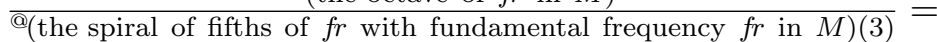

$\frac{32 \text { qua real number }}{27}$. The theorem is a consequence of (75).

Let $M$ be a space of music and $s_{10}$ be an element of (the carrier of $\left.M\right)^{2}$. We say that $s_{10}$ is monotonic if and only if

(Def. 54) there exists an element $f r$ of $M$ and there exist positive real numbers $r_{1}$, $r_{2}$ such that $s_{10}(1)=f r$ and $s_{10}(1)=r_{1}$ and $s_{10}(2)=r_{2}$ and $r_{1}<r_{2}$ and $s_{10}(2)=$ the octave of $f r$ in $M$.

Let $s_{10}$ be an element of (the carrier of $\left.M\right)^{3}$. We say that $s_{10}$ is ditonic if and only if

(Def. 55) there exists an element $f r$ of $M$ and there exist positive real numbers $r_{1}, r_{2}, r_{3}$ such that $s_{10}(1)=f r$ and $s_{10}(1)=r_{1}$ and $s_{10}(2)=r_{2}$ and $s_{10}(3)=r_{3}$ and $r_{1}<r_{2}<r_{3}$ and $s_{10}(3)=$ the octave of $f r$ in $M$.

Let $s_{10}$ be an element of (the carrier of $\left.M\right)^{4}$. We say that $s_{10}$ is tritonic if and only if

(Def. 56) there exists an element $f r$ of $M$ and there exist positive real numbers $r_{1}, r_{2}, r_{3}, r_{4}$ such that $s_{10}(1)=f r$ and $s_{10}(1)=r_{1}$ and $s_{10}(2)=r_{2}$ and $s_{10}(3)=r_{3}$ and $s_{10}(4)=r_{4}$ and $r_{1}<r_{2}<r_{3}$ and $r_{3}<r_{4}$ and $s_{10}(4)=$ the octave of $f r$ in $M$.

Let $s_{10}$ be an element of (the carrier of $\left.M\right)^{5}$. We say that $s_{10}$ is tetratonic if and only if

(Def. 57) there exists an element $f r$ of $M$ and there exist positive real numbers $r_{1}, r_{2}, r_{3}, r_{4}, r_{5}$ such that $s_{10}(1)=f r$ and $s_{10}(1)=r_{1}$ and $s_{10}(2)=r_{2}$ and $s_{10}(3)=r_{3}$ and $s_{10}(4)=r_{4}$ and $s_{10}(5)=r_{5}$ and $r_{1}<r_{2}<r_{3}$ and $r_{3}<r_{4}<r_{5}$ and $s_{10}(5)=$ the octave of $f r$ in $M$.

Let $n$ be a natural number and $s_{10}$ be an element of (the carrier of $\left.M\right)^{n}$. We say that $s_{10}$ is pentatonic if and only if

(Def. 58) $n=6$ and there exists an element $f r$ of $M$ and there exist positive real numbers $r_{1}, r_{2}, r_{3}, r_{4}, r_{5}, r_{6}$ such that $s_{10}(1)=f r$ and $s_{10}(1)=r_{1}$ and $s_{10}(2)=r_{2}$ and $s_{10}(3)=r_{3}$ and $s_{10}(4)=r_{4}$ and $s_{10}(5)=r_{5}$ and $s_{10}(6)=$ 
$r_{6}$ and $r_{1}<r_{2}<r_{3}$ and $r_{3}<r_{4}<r_{5}$ and $r_{5}<r_{6}$ and $s_{10}(6)=$ the octave of $f r$ in $M$.

Let $s_{10}$ be an element of (the carrier of $\left.M\right)^{7}$. We say that $s_{10}$ is hexatonic if and only if

(Def. 59) there exists an element $f r$ of $M$ and there exist positive real numbers $r_{1}$, $r_{2}, r_{3}, r_{4}, r_{5}, r_{6}, r_{7}$ such that $s_{10}(1)=f r$ and $s_{10}(1)=r_{1}$ and $s_{10}(2)=r_{2}$ and $s_{10}(3)=r_{3}$ and $s_{10}(4)=r_{4}$ and $s_{10}(5)=r_{5}$ and $s_{10}(6)=r_{6}$ and $s_{10}(7)=r_{7}$ and $r_{1}<r_{2}<r_{3}$ and $r_{3}<r_{4}<r_{5}$ and $r_{5}<r_{6}<r_{7}$ and $s_{10}(7)=$ the octave of $f r$ in $M$.

Let $n$ be a natural number and $s_{10}$ be an element of (the carrier of $\left.M\right)^{n}$. We say that $s_{10}$ is heptatonic if and only if

(Def. 60) $n=8$ and there exists an element fr of $M$ and there exist positive real numbers $r_{1}, r_{2}, r_{3}, r_{4}, r_{5}, r_{6}, r_{7}, r_{8}$ such that $s_{10}(1)=f r$ and $s_{10}(1)=r_{1}$ and $s_{10}(2)=r_{2}$ and $s_{10}(3)=r_{3}$ and $s_{10}(4)=r_{4}$ and $s_{10}(5)=r_{5}$ and $s_{10}(6)=r_{6}$ and $s_{10}(7)=r_{7}$ and $s_{10}(8)=r_{8}$ and $r_{1}<r_{2}<r_{3}$ and $r_{3}<r_{4}<r_{5}$ and $r_{5}<r_{6}<r_{7}$ and $r_{7}<r_{8}$ and $s_{10}(8)=$ the octave of $f r$ in $M$.

Let $s_{10}$ be an element of (the carrier of $\left.M\right)^{9}$. We say that $s_{10}$ is octatonic if and only if

(Def. 61) there exists an element $f r$ of $M$ and there exist positive real numbers $r_{1}, r_{2}, r_{3}, r_{4}, r_{5}, r_{6}, r_{7}, r_{8}, r_{9}$ such that $s_{10}(1)=f r$ and $s_{10}(1)=r_{1}$ and $s_{10}(2)=r_{2}$ and $s_{10}(3)=r_{3}$ and $s_{10}(4)=r_{4}$ and $s_{10}(5)=r_{5}$ and $s_{10}(6)=$ $r_{6}$ and $s_{10}(7)=r_{7}$ and $s_{10}(8)=r_{8}$ and $s_{10}(9)=r_{9}$ and $r_{1}<r_{2}<r_{3}$ and $r_{3}<r_{4}<r_{5}$ and $r_{5}<r_{6}<r_{7}$ and $r_{7}<r_{8}<r_{9}$ and $s_{10}(9)=$ the octave of $f r$ in $M$.

\section{Pentatonic Pythagorean Scale}

Let $M$ be a space of music and $f r$ be an element of $M$. The pentatonic pythagorean scale of $f r$ in $M$ yielding an element of (the carrier of $M)^{6}$ is defined by

(Def. 62) $i t(1)=f r$ and $i t(2)=($ the spiral of fifths of $f r$ with fundamental frequency $f r$ in $M)(2)$ and $i t(3)=($ the spiral of fifths of $f r$ with fundamental frequency $f r$ in $M)(4)$ and $i t(4)=$ (the spiral of fifths of $f r$ with fundamental frequency $f r$ in $M)(1)$ and it $(5)=($ the spiral of fifths of $f r$ with fundamental frequency $f r$ in $M)(3)$ and it $(6)=$ the octave of $f r$ in $M$.

From now on $M$ denotes a space of music and $f_{10}, f r, f_{1}, f_{2}$ denote elements of $M$.

Now we state the proposition: 
(83) The pentatonic pythagorean scale of $f r$ in $M$ is pentatonic. The theorem is a consequence of (74), (76), (73), and (75).

Let $M$ be a space of music and $f_{1}, f_{2}$ be elements of $M$. The interval between $f_{1}$ and $f_{2}$ yielding a positive real number is defined by

(Def. 63) there exist positive real numbers $r_{1}, r_{2}$ such that $r_{1}=f_{1}$ and $r_{2}=f_{2}$ and $i t=\frac{r_{2}}{r_{1}}$.

The pythagorean tone yielding a positive real number is defined by the term (Def. 64) $\frac{\text { (9 qua real number) }}{8}$.

The pythagorean semiditone yielding a positive real number is defined by the term

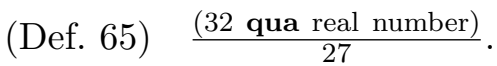

The pythagorean major third yielding a positive real number is defined by the term

(Def. 66) (the pythagorean tone) · (the pythagorean tone).

The pythagorean pure major third yielding a positive real number is defined by the term

(Def. 67) (5 qua real number)

The syntonic comma yielding a positive real number is defined by the term

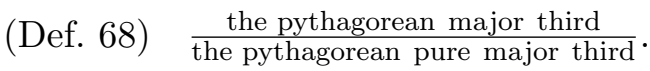

Now we state the propositions:

(84) The syntonic comma $=\frac{\text { (81 qua real number) }}{80}$.

(85) The pythagorean tone $<$ the pythagorean semiditone.

(86) (The pythagorean tone) $\cdot$ (the pythagorean tone) $\cdot($ the pythagorean semiditone $) \cdot($ the pythagorean tone $) \cdot($ the pythagorean semiditone $)=2$.

Let $M$ be a space of music and $f r$ be an element of $M$. The functors: the first degree of pentatonic scale of $f r$ in $M$, the second degree of pentatonic scale of $f r$ in $M$, the third degree of pentatonic scale of $f r$ in $M$, the fourth degree of pentatonic scale of $f r$ in $M$, and the fifth degree of pentatonic scale of $f r$ in $M$ yielding elements of $M$ are defined by terms

(Def. 69) (the pentatonic pythagorean scale of $f r$ in $M)(1)$,

(Def. 70) (the pentatonic pythagorean scale of $f r$ in $M)(2)$,

(Def. 71) (the pentatonic pythagorean scale of $f r$ in $M)(3)$,

(Def. 72) (the pentatonic pythagorean scale of $f r$ in $M$ )(4),

(Def. 73) (the pentatonic pythagorean scale of $f r$ in $M)(5)$, respectively. The octave of pentatonic scale of $f r$ in $M$ yielding an element of $M$ is defined by the term

(Def. 74) the octave of $f r$ in $M$. 
Now we state the propositions:

(87) There exist elements $r_{1}, r_{2}$ of $\mathbb{R}_{+}$such that the interval between $f_{1}$ and $f_{2}=\mathbb{R}$-ratio $\left(r_{1}, r_{2}\right)$.

(88) Let us consider positive real numbers $r_{1}, r_{2}, r_{3}, r_{4}, r_{5}, r_{6}$. Suppose (the pentatonic pythagorean scale of $f r$ in $M)(1)=r_{1}$ and (the pentatonic pythagorean scale of $f r$ in $M)(2)=r_{2}$ and (the pentatonic pythagorean scale of $f r$ in $M)(3)=r_{3}$ and (the pentatonic pythagorean scale of $f r$ in $M)(4)=r_{4}$ and (the pentatonic pythagorean scale of $f r$ in $\left.M\right)(5)=r_{5}$ and (the pentatonic pythagorean scale of $f r$ in $M)(6)=r_{6}$. Then

(i) $\frac{r_{2}}{r_{1}}=\frac{(9 \text { qua real number })}{8}$, and

(ii) $\frac{r_{3}}{r_{2}}=\frac{(9 \text { qua real number })}{8}$, and

(iii) $\frac{r_{4}}{r_{3}}=\frac{(32 \text { qua real number })}{27}$, and

(iv) $\frac{r_{5}}{r_{4}}=\frac{(9 \text { qua real number })}{8}$, and

(v) $\frac{r_{6}}{r_{5}}=\frac{(32 \text { qua real number })}{27}$.

The theorem is a consequence of (83), (78), (79), (80), (81), and (82).

(89) There exist positive real numbers $r_{1}, r_{2}, r_{3}, r_{4}, r_{5}, r_{6}$ such that

(i) (the pentatonic pythagorean scale of $f r$ in $M)(1)=r_{1}$, and

(ii) (the pentatonic pythagorean scale of $f r$ in $M)(2)=r_{2}$, and

(iii) (the pentatonic pythagorean scale of $f r$ in $M)(3)=r_{3}$, and

(iv) (the pentatonic pythagorean scale of $f r$ in $M)(4)=r_{4}$, and

(v) (the pentatonic pythagorean scale of $f r$ in $M)(5)=r_{5}$, and

(vi) (the pentatonic pythagorean scale of $f r$ in $M)(6)=r_{6}$, and

(vii) $\frac{r_{2}}{r_{1}}=\frac{(9 \text { qua real number })}{8}$, and

(viii) $\frac{r_{3}}{r_{2}}=\frac{(9 \text { qua real number })}{8}$, and

(ix) $\frac{r_{4}}{r_{3}}=\frac{(32 \text { qua real number })}{27}$, and

(x) $\frac{r_{5}}{r_{4}}=\frac{(9 \text { qua real number })}{8}$, and

(xi) $\frac{r_{6}}{r_{5}}=\frac{(32 \text { qua real number })}{27}$.

The theorem is a consequence of (1) and (88).

(90) $\frac{\text { (9 qua real number) }}{8}=\frac{\text { (9 qua rational number) }}{8}$.

(91) (i) the interval between the first degree of pentatonic scale of $f r$ in $M$ and (the second degree of pentatonic scale of $f r$ in $M$ ) $=$ the pythagorean tone, and 
(ii) the interval between the second degree of pentatonic scale of fr in $M$ and (the third degree of pentatonic scale of $f r$ in $M$ ) $=$ the pythagorean tone, and

(iii) the interval between the third degree of pentatonic scale of $f r$ in $M$ and (the fourth degree of pentatonic scale of $f r$ in $M$ ) = the pythagorean semiditone, and

(iv) the interval between the fourth degree of pentatonic scale of $f r$ in $M$ and (the fifth degree of pentatonic scale of $f r$ in $M$ ) $=$ the pythagorean tone, and

(v) the interval between the fifth degree of pentatonic scale of $f r$ in $M$ and (the octave of pentatonic scale of $f r$ in $M$ ) $=$ the pythagorean semiditone.

The theorem is a consequence of (89).

(92) the fifth of $f r$ in $M$ is between $f r$ and the octave of $f r$ in $M$.

Let us consider positive real numbers $r_{1}, r_{2}$. Now we state the propositions:

(93) Suppose $f_{1}=r_{1}$ and $f_{2}=r_{2}$ and $r_{2}=\frac{(4 \text { qua real number })}{3} \cdot r_{1}$. Then

(i) the fifth of $f_{2}$ in $M=2 \cdot r_{1}$, and

(ii) the fifth of $f_{2}$ in $M$ is not between $f_{1}$ and the octave of $f_{1}$ in $M$.

(94) Suppose $f_{1}=r_{1}$ and $f_{2}=r_{2}$ and $r_{2}=\frac{(4 \text { qua real number })}{3} \cdot r_{1}$. Then

(i) if the fifth of $f_{2}$ in $M$ is between $f_{10}$ and the octave of $f_{10}$ in $M$, then the octave descending of (the reduct fifth of the $f_{2}$ with fundamental frequency $f_{10}$ in $M$ ) in $M=f_{1}$, and

(ii) if the fifth of $f_{2}$ in $M$ is not between $f_{10}$ and the octave of $f_{10}$ in $M$, then the reduct fifth of the $f_{2}$ with fundamental frequency $f_{10}$ in $M=f_{1}$.

The theorem is a consequence of (62).

(95) Suppose $f_{1}=r_{1}$ and $f_{2}=r_{2}$ and $r_{2}=\frac{(4 \text { qua real number })}{3} \cdot r_{1}$. Then the reduct fifth of the $f_{2}$ with fundamental frequency $f_{1}$ in $M=f_{1}$. The theorem is a consequence of (94) and (93).

\section{Heptatonic Pythagorean Scale}

Let $S$ be a space of music. We say that $S$ is fourth constructible if and only if

(Def. 75) for every element $f r$ of $S$, there exists an element $q$ of $S$ such that $\langle f r$, $q\rangle \in$ the set of fourth of $S$. 
Now we state the propositions:

(96) Let us consider a space of music $M$. Suppose $M=\mathbb{R}$-music. Let us consider an element $f r$ of $M$. Then there exist positive real numbers $f, q_{1}$ such that

(i) $f=f r$, and

(ii) $q_{1}=\frac{(4 \text { qua real number })}{3} \cdot f$, and

(iii) $\left\langle f, q_{1}\right\rangle \in$ the set of fourth of $M$.

The theorem is a consequence of (1) and (24).

(97) $\mathbb{R}$-music is fourth constructible. The theorem is a consequence of (96) and (1).

One can verify that there exists a space of music which is fourth constructible.

Let $M$ be a fourth constructible space of music and fr be an element of $M$. The fourth of $f r$ in $M$ yielding an element of $M$ is defined by

(Def. 76) $\langle f r, i t\rangle \in$ the set of fourth of $M$.

We say that $M$ is classical fourth if and only if

(Def. 77) for every element $f r$ of $M$, there exists a positive real number $f$ such that $f r=f$ and the fourth of $f r$ in $M=\frac{(4 \text { qua real number })}{3} \cdot f$.

Now we state the proposition:

(98) Let us consider a fourth constructible space of music $M$. Suppose $M=$ $\mathbb{R}$-music. Let us consider an element fr of $M$. Then there exists a positive real number $f$ such that

(i) $f r=f$, and

(ii) the fourth of $f r$ in $M=\frac{(4 \text { qua real number })}{3} \cdot f$.

The theorem is a consequence of (1) and (96).

Let us note that there exists a fourth constructible space of music which is classical fourth.

Let $M$ be a satisfying real, non empty structure of music. We say that $M$ is euclidean if and only if

(Def. 78) for every elements $f_{1}, f_{2}$ of $M$, (the Ratio of $\left.M\right)\left(f_{1}, f_{2}\right)=\frac{{ }^{\circledR} f_{2}}{{ }^{@} f_{1}}$.

One can verify that there exists a satisfying real, non empty structure of music which is euclidean and every satisfying real, non empty structure of music which is euclidean is also satisfying interval and every satisfying real, non empty structure of music which is euclidean is also unison-ratio stable and every satisfying real, non empty structure of music which is euclidean is also ratio symmetric and there exists a classical fourth, fourth constructible space of music which is euclidean. 
A heptatonic pythagorean score is a classical fourth, fourth constructible space of music. From now on $H$ denotes a heptatonic pythagorean score and $f r$ denotes an element of $H$.

Let $H$ be a heptatonic pythagorean score and $f r$ be an element of $H$. The heptatonic pythagorean scale of $f r$ in $H$ yielding an element of (the carrier of $H)^{8}$ is defined by

(Def. 79) $\quad i t(1)=($ the spiral of fifths of (the fourth of $f r$ in $H$ ) with fundamental frequency $f r$ in $H)(1)$ and $i t(2)=$ (the spiral of fifths of (the fourth of $f r$ in $H$ ) with fundamental frequency $f r$ in $H)(3)$ and it(3) $=($ the spiral of fifths of (the fourth of $f r$ in $H$ ) with fundamental frequency $f r$ in $H)(5)$ and $i t(4)=$ the fourth of $f r$ in $H$ and $i t(5)=($ the spiral of fifths of (the fourth of $f r$ in $H$ ) with fundamental frequency $f r$ in $H)(2)$ and $i t(6)=($ the spiral of fifths of (the fourth of $f r$ in $H$ ) with fundamental frequency $f r$ in $H)(4)$ and $i t(7)=($ the spiral of fifths of (the fourth of $f r$ in $H$ ) with fundamental frequency $f r$ in $H)(6)$ and $i t(8)=$ the octave of (the spiral of fifths of (the fourth of $f r$ in $H$ ) with fundamental frequency fr in $H)(1)$ in $H$.

Now we state the propositions:

(99) the fourth of $f r$ in $H$ is between $f r$ and the octave of $f r$ in $H$.

(100) Let us consider a natural number $n$. Then (the spiral of fifths of (the fourth of $f r$ in $H$ ) with fundamental frequency $f r$ in $H)(n)$ is between $f r$ and the octave of $f r$ in $H$.

(101) (The spiral of fifths of (the fourth of $f r$ in $H$ ) with fundamental frequency $f r$ in $H)(1)=f r$. The theorem is a consequence of (66) and (62).

(102) (The spiral of fifths of (the fourth of $f r$ in $H$ ) with fundamental frequency $f r$ in $H)(2)=\frac{(3 \text { qua real number })}{2} \cdot\left({ }^{@} f r\right)$. The theorem is a consequence of (101) and (66).

(103) (The spiral of fifths of (the fourth of $f r$ in $H$ ) with fundamental frequency $f r$ in $H)(3)=\frac{(9 \text { qua real number })}{8} \cdot\left({ }^{@} f r\right)$. The theorem is a consequence of (102), (66), and (62).

(104) (The spiral of fifths of (the fourth of $f r$ in $H$ ) with fundamental frequency $f r$ in $H)(4)=\frac{(27 \text { qua real number })}{16} \cdot\left({ }^{\circledR} f r\right)$. The theorem is a consequence of (103) and (66).

(105) (The spiral of fifths of (the fourth of $f r$ in $H$ ) with fundamental frequency $f r$ in $H)(5)=\frac{(81 \text { qua real number })}{64} \cdot\left({ }^{\circledR} f r\right)$. The theorem is a consequence of (104), (66), and (62).

(106) (The spiral of fifths of (the fourth of $f r$ in $H$ ) with fundamental frequency $f r$ in $H)(6)=\frac{(243 \text { qua real number })}{128} \cdot\left({ }^{@} f r\right)$. The theorem is a consequence of 
(105) and (66).

(107) (i) (the heptatonic pythagorean scale of $f r$ in $H)(1)=1 \cdot\left({ }^{@} f r\right)$, and

(ii) (the heptatonic pythagorean scale of $f r$ in $H)(2)=\frac{(9 \text { qua real number })}{8}$. $\left({ }^{@} f r\right)$, and

(iii) (the heptatonic pythagorean scale of $f r$ in $H)(3)=\frac{\text { (81 qua real number) }}{64}$. $\left({ }^{@} f r\right)$, and

(iv) (the heptatonic pythagorean scale of $f r$ in $H)(4)=\frac{(4 \text { qua real number })}{3}$. $\left({ }^{@} f r\right)$, and

(v) (the heptatonic pythagorean scale of $f r$ in $H)(5)=\frac{(3 \text { qua real number })}{2}$. $\left({ }^{\circledR} f r\right)$, and

(vi) (the heptatonic pythagorean scale of $f r$ in $H)(6)=\frac{(27 \text { qua real number) }}{16}$. $\left({ }^{@} f r\right)$, and

(vii) (the heptatonic pythagorean scale of $f r$ in $H)(7)=\frac{(243 \text { qua real number) }}{128}$. $\left({ }^{@} f r\right)$, and

(viii) (the heptatonic pythagorean scale of $f r$ in $H)(8)=2 \cdot\left({ }^{@} f r\right)$.

The theorem is a consequence of (101), (103), (105), (102), (104), and (106).

(108) The heptatonic pythagorean scale of $f r$ in $H$ is heptatonic. The theorem is a consequence of (107).

The pythagorean semitone yielding a positive real number is defined by the term

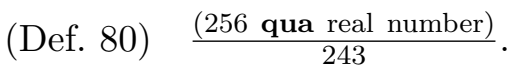

Now we state the propositions:

(109) $\frac{\text { the pythagorean tone }}{2}<$ the pythagorean semitone.

(110) (The pythagorean tone) · (the pythagorean tone) · (the pythagorean

semitone).(the pythagorean tone).(the pythagorean tone) $\cdot($ the pythagorean tone $) \cdot($ the pythagorean semitone $)=2$.

Let $H$ be a heptatonic pythagorean score and $f r$ be an element of $H$. The functors: the first degree of heptatonic scale of $f r$ in $H$, the second degree of heptatonic scale of $f r$ in $H$, the third degree of heptatonic scale of $f r$ in $H$, the fourth degree of heptatonic scale of $f r$ in $H$, and the fifth degree of heptatonic scale of $f r$ in $H$ yielding elements of $H$ are defined by terms

(Def. 81) (the heptatonic pythagorean scale of $f r$ in $H$ )(1),

(Def. 82) (the heptatonic pythagorean scale of $f r$ in $H$ )(2),

(Def. 83) (the heptatonic pythagorean scale of $f r$ in $H)(3)$,

(Def. 84) (the heptatonic pythagorean scale of $f r$ in $H$ )(4), 
(Def. 85) (the heptatonic pythagorean scale of $f r$ in $H)(5)$, respectively. The functors: the sixth degree of heptatonic scale of $f r$ in $H$, the seventh degree of heptatonic scale of $f r$ in $H$, and the eight degree of heptatonic scale of $f r$ in $H$ yielding elements of $H$ are defined by terms

(Def. 86) (the heptatonic pythagorean scale of $f r$ in $H)(6)$,

(Def. 87) (the heptatonic pythagorean scale of $f r$ in $H)(7)$,

(Def. 88) the octave of $f r$ in $H$, respectively. Now we state the proposition:

(111) (i) the interval between the first degree of heptatonic scale of $f r$ in $H$ and (the second degree of heptatonic scale of $f r$ in $H$ ) $=$ the pythagorean tone, and

(ii) the interval between the second degree of heptatonic scale of $f r$ in $H$ and (the third degree of heptatonic scale of $f r$ in $H$ ) $=$ the pythagorean tone, and

(iii) the interval between the third degree of heptatonic scale of $f r$ in $H$ and (the fourth degree of heptatonic scale of $f r$ in $H$ ) $=$ the pythagorean semitone, and

(iv) the interval between the fourth degree of heptatonic scale of $f r$ in $H$ and (the fifth degree of heptatonic scale of $f r$ in $H$ ) $=$ the pythagorean tone, and

(v) the interval between the fifth degree of heptatonic scale of $f r$ in $H$ and (the sixth degree of heptatonic scale of $f r$ in $H$ ) $=$ the pythagorean tone, and

(vi) the interval between the sixth degree of heptatonic scale of $f r$ in $H$ and (the seventh degree of heptatonic scale of $f r$ in $H$ ) $=$ the pythagorean tone, and

(vii) the interval between the seventh degree of heptatonic scale of $f r$ in $H$ and (the eight degree of heptatonic scale of $f r$ in $H$ ) $=$ the pythagorean semitone.

The theorem is a consequence of (107).

From now on $H$ denotes a heptatonic pythagorean score and $f r$ denotes an element of $H$.

Let $M$ be a space of music, $n$ be a natural number, and $s_{10}$ be an element of (the carrier of $M)^{n}$. Assume $s_{10}$ is heptatonic. We say that $s_{10}$ is perfect fifth if and only if

(Def. 89) $\left\langle s_{10}(1), s_{10}(5)\right\rangle,\left\langle s_{10}(2), s_{10}(6)\right\rangle,\left\langle s_{10}(3), s_{10}(7)\right\rangle,\left\langle s_{10}(4), s_{10}(8)\right\rangle \in$ the set of fifth of $M$. 
Now we state the proposition:

(112) Let us consider an euclidean heptatonic pythagorean score $H$, and an element $f r$ of $H$. Then the heptatonic pythagorean scale of $f r$ in $H$ is perfect fifth. The theorem is a consequence of (108), (107), and (24).

Let $H$ be a heptatonic pythagorean score and $f r$ be an element of $H$. The heptatonic pythagorean scale ascending of $f r$ in $H$ yielding an element of (the carrier of $H)^{8}$ is defined by the term

(Def. 90) the heptatonic pythagorean scale of (the octave of $f r$ in $H$ ) in $H$.

Now we state the propositions:

(113) (i) (the heptatonic pythagorean scale ascending of $f r$ in $H)(1)=2$. ( $\left.{ }^{@} f r\right)$, and

(ii) (the heptatonic pythagorean scale ascending of $f r$ in $H)(2)=$ $\frac{9 \text { qua real number }}{4} \cdot\left({ }^{@} f r\right)$, and

(iii) (the heptatonic pythagorean scale ascending of $f r$ in $H)(3)=$ $\frac{81 \text { qua real number }}{32} \cdot\left({ }^{@} f r\right)$, and

(iv) (the heptatonic pythagorean scale ascending of $f r$ in $H)(4)=$ $\frac{8 \text { qua real number }}{3} \cdot\left({ }^{@} f r\right)$, and

(v) (the heptatonic pythagorean scale ascending of $f r$ in $H)(5)=$ (3 qua real number) · $\left({ }^{@} f r\right)$, and

(vi) (the heptatonic pythagorean scale ascending of $f r$ in $H)(6)=$ $\frac{27 \text { qua real number }}{8} \cdot\left({ }^{@} f r\right)$, and

(vii) (the heptatonic pythagorean scale ascending of $f r$ in $H)(7)=$ $\frac{243 \text { qua real number }}{64} \cdot\left({ }^{@} f r\right)$, and

(viii) (the heptatonic pythagorean scale ascending of $f r$ in $H)(8)=4 \cdot\left({ }^{@} f r\right)$.

The theorem is a consequence of (107).

(114) (The heptatonic pythagorean scale of $f r$ in $H)(8)=$ (the heptatonic pythagorean scale ascending of $f r$ in $H)(1)$. The theorem is a consequence of (107) and (113).

(115) (i) the interval between the fifth degree of heptatonic scale of $f r$ in $H$ and (the second degree of heptatonic scale of (the octave of $f r$ in $H$ ) in $H)=\frac{(3 \text { qua real number })}{2}$, and

(ii) the interval between the sixth degree of heptatonic scale of $f r$ in $H$ and (the third degree of heptatonic scale of (the octave of $f r$ in $H$ ) in $H)=\frac{(3 \text { qua real number })}{2}$, and

(iii) the interval between the seventh degree of heptatonic scale of $f r$ in $H$ and (the fourth degree of heptatonic scale of (the octave of $f r$ in $H)$ in $H) \neq \frac{(3 \text { qua real number })}{2}$, and 
(iv) the interval between the eight degree of heptatonic scale of $f r$ in $H$ and (the fifth degree of heptatonic scale of (the octave of $\mathrm{fr}$ in $\mathrm{H}$ ) in $H)=\frac{(3 \text { qua real number })}{2}$.

The theorem is a consequence of (107) and (113).

(116) Let us consider an euclidean heptatonic pythagorean score $H$, and elements $f_{1}, f_{2}$ of $H$. Then the interval between $f_{1}$ and $f_{2}=$ (the Ratio of $H)\left(f_{1}, f_{2}\right)$.

(117) Let us consider an euclidean heptatonic pythagorean score $H$, and an element fr of $H$. Then

(i) $\langle($ the heptatonic pythagorean scale of $f r$ in $H)(5)$, (the heptatonic pythagorean scale ascending of $f r$ in $H)(2)\rangle$, 〈(the heptatonic pythagorean scale of $f r$ in $H)(6)$, (the heptatonic pythagorean scale ascending of $f r$ in $H)(3)\rangle \in$ the set of fifth of $H$, and

(ii) $\langle($ the heptatonic pythagorean scale of $f r$ in $H)(7)$, (the heptatonic pythagorean scale ascending of $f r$ in $H)(4)\rangle \notin$ the set of fifth of $H$.

The theorem is a consequence of (115), (24), and (116).

Let $H$ be a space of music, $n$ be a non zero, natural number, $s_{10}$ be an element of (the carrier of $H)^{n}$, and $i$ be a natural number. The functor $\#_{i}^{s_{10}}$ yielding an element of $H$ is defined by the term

(Def. 91) $\begin{cases}s_{10}(i), & \text { if } i \in \operatorname{Seg} n, \\ \text { the element of } H, & \text { otherwise. }\end{cases}$

Assume $s_{10}$ is heptatonic. We say that $s_{10}$ is dorian if and only if

(Def. 92) there exist positive real numbers $t_{1}, t_{2}$ such that $t_{1} \cdot t_{1} \cdot t_{1} \cdot t_{1} \cdot t_{1} \cdot t_{2}$. $t_{2}=2$ and the interval between $\#_{1}^{s_{10}}$ and $\#_{2}^{s_{10}}=t_{1}$ and the interval between $\#_{2}^{s_{10}}$ and $\#_{3}^{s_{10}}=t_{2}$ and the interval between $\#_{3}^{s_{10}}$ and $\#_{4}^{s_{10}}=t_{1}$ and the interval between $\#_{4}^{s_{10}}$ and $\#_{5}^{s_{10}}=t_{1}$ and the interval between $\#_{5}^{s_{10}}$ and $\#_{6}^{s_{10}}=t_{1}$ and the interval between $\#_{6}^{s_{10}}$ and $\#_{7}^{s_{10}}=t_{2}$ and the interval between $\#_{7}^{s_{10}}$ and $\#_{8}^{s_{10}}=t_{1}$.

Assume $s_{10}$ is heptatonic. We say that $s_{10}$ is hypodorian if and only if

(Def. 93) there exist positive real numbers $t_{1}, t_{2}$ such that $t_{1} \cdot t_{1} \cdot t_{1} \cdot t_{1} \cdot t_{1} \cdot t_{2}$. $t_{2}=2$ and the interval between $\#_{1}^{s_{10}}$ and $\#_{2}^{s_{10}}=t_{1}$ and the interval between $\#_{2}^{s_{10}}$ and $\#_{3}^{s_{10}}=t_{2}$ and the interval between $\#_{3}^{s_{10}}$ and $\#_{4}^{s_{10}}=t_{1}$ and the interval between $\#_{4}^{s_{10}}$ and $\#_{5}^{s_{10}}=t_{1}$ and the interval between $\#_{5}^{s_{10}}$ and $\#_{6}^{s_{10}}=t_{2}$ and the interval between $\#_{6}^{s_{10}}$ and $\#_{7}^{s_{10}}=t_{1}$ and the interval between $\#_{7}^{s_{10}}$ and $\#_{8}^{s_{10}}=t_{1}$.

Assume $s_{10}$ is heptatonic. We say that $s_{10}$ is phrygian if and only if

(Def. 94) there exist positive real numbers $t_{1}, t_{2}$ such that $t_{1} \cdot t_{1} \cdot t_{1} \cdot t_{1} \cdot t_{1} \cdot t_{2}$. $t_{2}=2$ and the interval between $\#_{1}^{s_{10}}$ and $\#_{2}^{s_{10}}=t_{1}$ and the interval 
between $\#_{2}^{s_{10}}$ and $\#_{3}^{s_{10}}=t_{2}$ and the interval between $\#_{3}^{s_{10}}$ and $\#_{4}^{s_{10}}=t_{1}$ and the interval between $\#_{4}^{s_{10}}$ and $\#_{5}^{s_{10}}=t_{2}$ and the interval between $\#_{5}^{s 10}$ and $\#_{6}^{s 10}=t_{1}$ and the interval between $\#_{6}^{s_{10}}$ and $\#_{7}^{s_{10}}=t_{1}$ and the interval between $\#_{7}^{s_{10}}$ and $\#_{8}^{s_{10}}=t_{1}$.

Assume $s_{10}$ is heptatonic. We say that $s_{10}$ is hypophrygian if and only if

(Def. 95) there exist positive real numbers $t_{1}, t_{2}$ such that $t_{1} \cdot t_{1} \cdot t_{1} \cdot t_{1} \cdot t_{1} \cdot t_{2}$. $t_{2}=2$ and the interval between $\#_{1}^{s_{10}}$ and $\#_{2}^{s_{10}}=t_{2}$ and the interval between $\#_{2}^{s_{10}}$ and $\#_{3}^{s_{10}}=t_{1}$ and the interval between $\#_{3}^{s_{10}}$ and $\#_{4}^{s_{10}}=t_{1}$ and the interval between $\#_{4}^{s_{10}}$ and $\#_{5}^{s_{10}}=t_{2}$ and the interval between $\#_{5}^{s 10}$ and $\#_{6}^{s 10}=t_{1}$ and the interval between $\#_{6}^{s_{10}}$ and $\#_{7}^{s 10}=t_{1}$ and the interval between $\#_{7}^{s_{10}}$ and $\#_{8}^{s_{10}}=t_{1}$.

Assume $s_{10}$ is heptatonic. We say that $s_{10}$ is lydian if and only if

(Def. 96) there exist positive real numbers $t_{1}, t_{2}$ such that $t_{1} \cdot t_{1} \cdot t_{1} \cdot t_{1} \cdot t_{1} \cdot t_{2}$. $t_{2}=2$ and the interval between $\#_{1}^{s_{10}}$ and $\#_{2}^{s_{10}}=t_{1}$ and the interval between $\#_{2}^{s_{10}}$ and $\#_{3}^{s_{10}}=t_{1}$ and the interval between $\#_{3}^{s_{10}}$ and $\#_{4}^{s_{10}}=t_{2}$ and the interval between $\#_{4}^{s_{10}}$ and $\#_{5}^{s_{10}}=t_{1}$ and the interval between $\#_{5}^{s 10}$ and $\#_{6}^{s_{10}}=t_{1}$ and the interval between $\#_{6}^{s_{10}}$ and $\#_{7}^{s_{10}}=t_{2}$ and the interval between $\#_{7}^{s_{10}}$ and $\#_{8}^{s_{10}}=t_{1}$.

Assume $s_{10}$ is heptatonic. We say that $s_{10}$ is hypolydian if and only if

(Def. 97) there exist positive real numbers $t_{1}, t_{2}$ such that $t_{1} \cdot t_{1} \cdot t_{1} \cdot t_{1} \cdot t_{1} \cdot t_{2}$. $t_{2}=2$ and the interval between $\#_{1}^{s_{10}}$ and $\#_{2}^{s_{10}}=t_{1}$ and the interval between $\#_{2}^{s_{10}}$ and $\#_{3}^{s_{10}}=t_{1}$ and the interval between $\#_{3}^{s_{10}}$ and $\#_{4}^{s_{10}}=t_{2}$ and the interval between $\#_{4}^{s_{10}}$ and $\#_{5}^{s_{10}}=t_{1}$ and the interval between $\#_{5}^{s_{10}}$ and $\#_{6}^{s_{10}}=t_{1}$ and the interval between $\#_{6}^{s_{10}}$ and $\#_{7}^{s_{10}}=t_{1}$ and the interval between $\#_{7}^{s_{10}}$ and $\#_{8}^{s_{10}}=t_{2}$.

Assume $s_{10}$ is heptatonic. We say that $s_{10}$ is mixolydian if and only if

(Def. 98) there exist positive real numbers $t_{1}, t_{2}$ such that $t_{1} \cdot t_{1} \cdot t_{1} \cdot t_{1} \cdot t_{1} \cdot t_{2}$. $t_{2}=2$ and the interval between $\#_{1}^{s_{10}}$ and $\#_{2}^{s_{10}}=t_{1}$ and the interval between $\#_{2}^{s_{10}}$ and $\#_{3}^{s_{10}}=t_{1}$ and the interval between $\#_{3}^{s_{10}}$ and $\#_{4}^{s_{10}}=t_{2}$ and the interval between $\#_{4}^{s_{10}}$ and $\#_{5}^{s_{10}}=t_{1}$ and the interval between $\#_{5}^{s_{10}}$ and $\#_{6}^{s_{10}}=t_{1}$ and the interval between $\#_{6}^{s_{10}}$ and $\#_{7}^{s_{10}}=t_{2}$ and the interval between $\#_{7}^{s_{10}}$ and $\#_{8}^{s_{10}}=t_{1}$.

Assume $s_{10}$ is heptatonic. We say that $s_{10}$ is hypomixolydian if and only if (Def. 99) there exist positive real numbers $t_{1}, t_{2}$ such that $t_{1} \cdot t_{1} \cdot t_{1} \cdot t_{1} \cdot t_{1} \cdot t_{2}$. $t_{2}=2$ and the interval between $\#_{1}^{s 10}$ and $\#_{2}^{s_{10}}=t_{1}$ and the interval between $\#_{2}^{s_{10}}$ and $\#_{3}^{s_{10}}=t_{2}$ and the interval between $\#_{3}^{s_{10}}$ and $\#_{4}^{s_{10}}=t_{1}$ and the interval between $\#_{4}^{s_{10}}$ and $\#_{5}^{s_{10}}=t_{1}$ and the interval between $\#_{5}^{s_{10}}$ and $\#_{6}^{s_{10}}=t_{1}$ and the interval between $\#_{6}^{s_{10}}$ and $\#_{7}^{s_{10}}=t_{2}$ and the interval between $\#_{7}^{s_{10}}$ and $\#_{8}^{s_{10}}=t_{1}$. 
Assume $s_{10}$ is heptatonic. We say that $s_{10}$ is eolian if and only if

(Def. 100) there exist positive real numbers $t_{1}, t_{2}$ such that $t_{1} \cdot t_{1} \cdot t_{1} \cdot t_{1} \cdot t_{1} \cdot t_{2}$. $t_{2}=2$ and the interval between $\#_{1}^{s_{10}}$ and $\#_{2}^{s_{10}}=t_{1}$ and the interval between $\#_{2}^{s_{10}}$ and $\#_{3}^{s_{10}}=t_{2}$ and the interval between $\#_{3}^{s_{10}}$ and $\#_{4}^{s_{10}}=t_{1}$ and the interval between $\#_{4}^{s_{10}}$ and $\#_{5}^{s_{10}}=t_{1}$ and the interval between $\#_{5}^{s 10}$ and $\#_{6}^{s_{10}}=t_{2}$ and the interval between $\#_{6}^{s_{10}}$ and $\#_{7}^{s_{10}}=t_{1}$ and the interval between $\#_{7}^{s_{10}}$ and $\#_{8}^{s_{10}}=t_{1}$.

Assume $s_{10}$ is heptatonic. We say that $s_{10}$ is hypoeolian if and only if

(Def. 101) there exist positive real numbers $t_{1}, t_{2}$ such that $t_{1} \cdot t_{1} \cdot t_{1} \cdot t_{1} \cdot t_{1} \cdot t_{2}$. $t_{2}=2$ and the interval between $\#_{1}^{s_{10}}$ and $\#_{2}^{s_{10}}=t_{2}$ and the interval between $\#_{2}^{s_{10}}$ and $\#_{3}^{s_{10}}=t_{1}$ and the interval between $\#_{3}^{s_{10}}$ and $\#_{4}^{s_{10}}=t_{1}$ and the interval between $\#_{4}^{s_{10}}$ and $\#_{5}^{s_{10}}=t_{1}$ and the interval between $\#_{5}^{s 10}$ and $\#_{6}^{s_{10}}=t_{2}$ and the interval between $\#_{6}^{s_{10}}$ and $\#_{7}^{s_{10}}=t_{1}$ and the interval between $\#_{7}^{s_{10}}$ and $\#_{8}^{s_{10}}=t_{1}$.

Assume $s_{10}$ is heptatonic. We say that $s_{10}$ is ionan if and only if

(Def. 102) there exist positive real numbers $t_{1}, t_{2}$ such that $t_{1} \cdot t_{1} \cdot t_{1} \cdot t_{1} \cdot t_{1} \cdot t_{2}$. $t_{2}=2$ and the interval between $\#_{1}^{s_{10}}$ and $\#_{2}^{s_{10}}=t_{1}$ and the interval between $\#_{2}^{s_{10}}$ and $\#_{3}^{s_{10}}=t_{1}$ and the interval between $\#_{3}^{s_{10}}$ and $\#_{4}^{s_{10}}=t_{2}$ and the interval between $\#_{4}^{s_{10}}$ and $\#_{5}^{s_{10}}=t_{1}$ and the interval between $\#_{5}^{s_{10}}$ and $\#_{6}^{s_{10}}=t_{1}$ and the interval between $\#_{6}^{s_{10}}$ and $\#_{7}^{s_{10}}=t_{1}$ and the interval between $\#_{7}^{s_{10}}$ and $\#_{8}^{s_{10}}=t_{2}$.

Assume $s_{10}$ is heptatonic. We say that $s_{10}$ is hypoionan if and only if

(Def. 103) there exist positive real numbers $t_{1}, t_{2}$ such that $t_{1} \cdot t_{1} \cdot t_{1} \cdot t_{1} \cdot t_{1} \cdot t_{2}$. $t_{2}=2$ and the interval between $\#_{1}^{s_{10}}$ and $\#_{2}^{s_{10}}=t_{1}$ and the interval between $\#_{2}^{s_{10}}$ and $\#_{3}^{s_{10}}=t_{1}$ and the interval between $\#_{3}^{s_{10}}$ and $\#_{4}^{s_{10}}=t_{2}$ and the interval between $\#_{4}^{s_{10}}$ and $\#_{5}^{s_{10}}=t_{1}$ and the interval between $\#_{5}^{s_{10}}$ and $\#_{6}^{s_{10}}=t_{1}$ and the interval between $\#_{6}^{s_{10}}$ and $\#_{7}^{s_{10}}=t_{2}$ and the interval between $\#_{7}^{s_{10}}$ and $\#_{8}^{s_{10}}=t_{1}$.

Now we state the proposition:

(118) The heptatonic pythagorean scale of $f r$ in $H$ is ionan. The theorem is a consequence of (108), (107), and (111).

Acknowledgement: I would like to thank Hélène Cambier (Professor of music history at the Music Academy La Louvière) and Marie Barbier (musical instrument: Recorder) for valuable suggestions. 


\section{REFERENCES}

[1] Grzegorz Bancerek. On the structure of Mizar types. In Herman Geuvers and Fairouz Kamareddine, editors, Electronic Notes in Theoretical Computer Science, volume 85, pages 69-85. Elsevier, 2003.

[2] Grzegorz Bancerek. The fundamental properties of natural numbers Formalized Mathematics, 1(1):41-46, 1990.

[3] Grzegorz Bancerek, Czesław Byliński, Adam Grabowski, Artur Korniłowicz, Roman Matuszewski, Adam Naumowicz, Karol Pąk, and Josef Urban. Mizar: State-of-the-art and beyond. In Manfred Kerber, Jacques Carette, Cezary Kaliszyk, Florian Rabe, and Volker Sorge, editors, Intelligent Computer Mathematics, volume 9150 of Lecture Notes in Computer Science, pages 261-279. Springer International Publishing, 2015. ISBN 978-3319-20614-1. doi 10.1007/978-3-319-20615-8_17.

[4] Grzegorz Bancerek, Czesław Byliński, Adam Grabowski, Artur Korniłowicz, Roman Matuszewski, Adam Naumowicz, and Karol Pak. The role of the Mizar Mathematical Library for interactive proof development in Mizar. Journal of Automated Reasoning, 61(1):9-32, 2018. do1:10.1007/s10817-017-9440-6

[5] François Baskevitch. Les représentations de la propagation du son, d'Aristote à l'Encyclopédie. PhD thesis, Université de Nantes, France, 2008.

[6] Adam Grabowski, Artur Korniłowicz, and Christoph Schwarzweller. On algebraic hierarchies in mathematical repository of Mizar. In M. Ganzha, L. Maciaszek, and M. Paprzycki, editors, Proceedings of the 2016 Federated Conference on Computer Science and Information Systems (FedCSIS), volume 8 of Annals of Computer Science and Information Systems, pages 363-371, 2016. doi 10.15439/2016F520.

[7] Bernard Parzysz and Yves Hellegouarch. Musique et mathématique: (suivi de) Gammes naturelles. Number 53. Association des Professeurs de Mathématiques de l'Enseignement Public (APMEP), Paris, 1984. 